\title{
Bifurcation Analysis of Toxoplasmosis Epidemic Control on Increased Controlled Rate of Suppressing the Rate of Infected Births
}

\author{
Meri Hari Yanni and Zulfahmi
}

\begin{abstract}
The toxoplasmosis epidemic is an infectious disease caused by the parasitic Toxoplasma Gondii. This disease attacks the human immune system and other organs in the body, resulting in damage to tissues. The spread of the disease is carried out in various ways, one of them is eating foods that are less hygienic or not cooked properly, resulting in parasites remain active. Provision of controlled therapy is one solution in controlling the epidemic against suppression of the birth rate infected with toxoplasmosis. This study discusses the bifurcation analysis of a mathematical model for controlling the toxoplasmosis epidemic. Bifurcation analysis is carried out on the controlled rate and rate of birth control of toxoplasmosis. From the mathematical model of controlling the toxoplasmosis epidemic, stability and existence analysis are performed at each equilibrium point. Next, a function of two independent parameters is constructed which influences the spread of the disease, namely the controlled rate and the rate of infected births. Then, a bifurcation analysis of each region is obtained from each function of the two free parameters. From the bifurcation analysis, three regional conditions were obtained which showed the dynamics of the toxoplasmosis epidemic of two independent parameters with each interpretation of the bifurcation region.
\end{abstract}

Index Terms-Stability and existence analysis, bifurcation analysis, interpretation of bifurcation analysis for each region.

\section{INTRODUCTION}

$\mathbf{T}$ OXOPLASMOSIS is a disease caused by the parasitic toxoplasma gondi, with the main source of its development being felidae animals. Parasites produced from cat feces that have sporulated in a free environment [1]. While in humans, and mammals as intermediaries host in the development of parasites. The spread of disease occurs through a variety of ways, one of which is the habit of eating food without washing hands, then eating fruits and vegetables that are less clean washed and eating foods that are undercooked.

The spread of Toxoplasmosis disease can occur worldwide. In Colombia, the research has been conducted using hybrid model of the spread of Toxoplasmosis between two countries [2]. The research focuses on the disease in the initial condition, during and after the diagnosis and treatment to reduce the potential of other individuals affected by the disease so that the strategy for public awareness in consuming healty food and drink and direct attention to the cat subpopulation. Furthermore, in Indonesia at the year 2010 reported that the number of prevalence rate of toxoplasmosis in the community in Banda

Manuscript received October 3, 2019; accepted December 7, 2019.

The authors are with STKIP Bumi Persada Lhokseumawe, Indonesia. Email: merihyanni@gmail.com
Aceh is $3.15 \%$ [3]. The habits of the people in Aceh city is the habit of eating satay and grilled chicken that is undercooked. These habits can have a harmful impact on the people of Aceh, including neurological disorders such as the brain, muscles and eyes, causing Alzheimer's and schizophrenia [4]. Whereas in pregnant women resulting in abortion, premature death in the fetus, and abnormalities in the baby. So that further socialization and analysis is needed regarding the spread of toxoplasmosis in the Lhokseumawe city community to reduce the number of individuals infected with toxoplasmosis in the future.

Research related to the spread of toxoplasmosis has been done and will continue to be developed with various perspectives and problems that occur in the community. Researchers have also conducted research that discusses several conditions that greatly affect the spread of toxoplasma gondi in the human body [5]. The study resulted in several conditions affecting the spread of disease in the human body with an indirect interaction by the definitive host.This research is also strengthened by research on the dynamics of the mathematical model of toxoplasmosis [6], which is then developed by taking into account interactions that occur in humans with cats in a region [7], [8] and the dynamics of the model of the spread of toxoplasmosis from spools of cats that have been sporulated [9]. Research on controlling the toxoplasmosis epidemic with case studies in the city of Lhokseumawe has been conducted [10]. In this research, researchers are developing on bifurcation analysis was carried out by releasing two parameters that affect the epidemic, so that the behavior obtained from the dynamics of the toxoplasmosis epidemic from various regional conditions.

The purpose of this study was to look at the dynamics of the toxoplasmosis epidemic through increasing the controlled rate by suppressing the rate of births infected with toxoplasmosis. Bifurcation analysis is performed to determine the behavior of the toxoplasmosis epidemic from each condition of the formed bifurcation area. Next, an interpretation of each bifurcation area was carried out which described the dynamics of the spread of toxoplasmosis in the Lhokseumawe city area.

\section{Methods}

This research is a theoretical and applied research study. Theoretical studies are carried out through a review of biological and medical literature relating to the epidemic of toxoplasmosis. Literature review is used in the formation of 


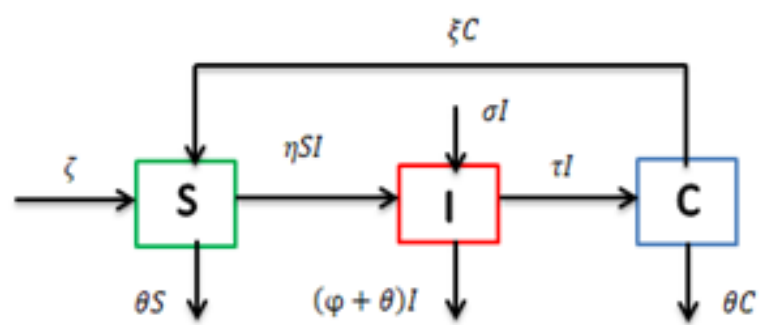

Fig. 1: Transfer diagram of the toxoplasmosis epidemic model.

mathematical models that first construct concepts in accordance with the facts of the epidemic. This study also conducted interviews with speakers, namely SpOG specialists related to toxoplasmosis. The data used in this study are secondary data on the number of patients with toxoplasmosis in 2018 at the hospital Cut Mutia, and other supporting data were also obtained from the Lhokseumawe City Health Office in 2018.

The first stage in this research is constructing a mathematical model of the toxoplasmosis epidemic in Lhokseumawe City. In constructing a mathematical model, the assumptions and criteria in the research variables were then tested and revised through interviewees. Next, analyze mathematical models analytically and numerically. The analysis was performed by determining the equilibrium point of the mathematical model, determining the stability criteria of the mathematical model, determining the basic reproduction numbers and determining the bifurcation limits of the occurrence of the toxoplasmosis epidemic. In the next stage, numerical simulations were conducted based on patient data that had been collected from the Hospital and the Health Service. Simulations were carried out to see the dynamics of the toxoplasmosis epidemic from each region with two parameters that influence the epidemic. Then proceed with interpreting the dynamics that appear in each region.

\section{MODEL FORMULATION}

\section{A. Construction of the Toxoplasmosis Epidemic Model}

The assumptions used in the formation of a mathematical model of the toxoplasmosis epidemic, are as follows:

1) In vulnerable populations, transmission occurs / spread horizontally.

2) Treatment is given to actively infected individuals.

3) When the immune system goes down, it allows vulnerable individuals to regain control.

4) Mothers infected with Toxoplasmosis can give birth to babies infected with Toxoplasmosis.

The mathematical model of the toxoplasmosis epidemic is represented in the following transfer diagram:

From Fig. 1, it can be seen that the human population is divided into SIC subpopulations. Interactions between the three populations in the toxoplasmosis epidemic model are presented in the form of an unusual differential equation as follows:

$$
\frac{d S}{d t}=\zeta-\theta S-\eta S I+\xi C
$$

$$
\begin{aligned}
\frac{d I}{d t} & =\eta S I-(\varphi+\theta) I+\sigma I-\tau I, \\
\frac{d C}{d t} & =\tau-\theta C-\xi C .
\end{aligned}
$$

The mathematical model above is expressed in the form of a non-linear differential equation, which satisfies the initial conditions of each variable is positive and each parameter in the System (1) is positive, $S(0) \geq 0, I(0) \geq 0$, and $C(0) \geq 0$, with the parameters used as follows:

- $\zeta=$ rate of birth,

- $\theta=$ natural death rate,

- $\eta=$ toxoplasmosis infection rate,

- $\xi=$ the rate of controlled individuals becoming vulnerable individuals,

- $\varphi=$ rate of death due to Toxoplasmosis,

- $\tau=$ controlled individual rate,

- $\sigma=$ the rate of birth that has been infected with Toxoplasma.

\section{B. Equilibrium Points and Basic Reproduction Numbers}

The Equilibrium Point in the System (1) is obtained by solving the equation $\frac{d S}{d t}=\frac{d I}{d t}=\frac{d C}{d t}=0$ so that it produces two equilibrium points namely the equilibrium point when there is no spread of toxoplasmosis in the community $q_{0}=\left(\frac{\zeta}{\theta}, 0,0\right)$ and the point of equilibrium when the spread of toxoplasmosis in the community $q_{1}=\left(S^{*}, I^{*}, C^{*}\right)$, where $S^{*}=\frac{\varphi+\tau+\theta-\sigma}{\eta}, I^{*}=$ $\frac{\zeta(\theta+\xi)-\theta S^{*}(\theta+\xi)}{\eta S^{*}(\theta+\xi)-\xi \tau}, C^{*}=\tau\left(\frac{\zeta-\theta S^{*}}{\eta S^{*}(\theta+\xi)-\xi \tau}\right)$.

Theorem 1: If $\varphi+\tau+\theta>\sigma, \zeta(\theta+\xi)>\theta S^{*}(\theta+\xi)$, $\eta S^{*}(\theta+\xi)>\xi \tau$ and $\zeta-\theta S^{*}>0$, then the endemic equilibrium point $q_{1}$ exists.

The basic reproduction number $\left(R_{0}\right)$ in this study uses the next generation operator approach method. First is the grouping of sub populations from (1) into compartments $X$, $Y$ and $Z$ :

$$
\begin{aligned}
& \frac{d X}{d t}=\left[\begin{array}{c}
\zeta-\theta S-\eta S I+\xi C \\
\tau-\theta C-\xi C
\end{array}\right]=f(X, Y, Z), \\
& \frac{d Y}{d t}=0=g(X, Y, Z), \\
& \frac{d Z}{d t}=\eta S I-(\varphi+\theta) I+\sigma I-\tau I=k(X, Y, Z) .
\end{aligned}
$$

Then the functions of $k(X, Y, Z)$ are derived from $I$, so that we obtain

$$
\frac{d k}{d t}\left(X^{*}, g\left(X^{*}, Z\right), Z\right)=\eta S-(\varphi+\theta)+\sigma-\tau
$$

Disease-free equilibrium point $q_{0}=\left(\frac{\zeta}{\theta}, 0,0\right)$, so (3) becomes

$$
\begin{aligned}
A & =\eta \frac{\zeta}{\theta}-(\varphi+\theta)+\sigma-\tau \\
& =\left(\eta \frac{\zeta}{\theta}+\sigma\right)-(\varphi+\theta+\tau)
\end{aligned}
$$

The basic reproduction number $R_{0}=M D^{-1}$, with $M=\eta \frac{\zeta}{\theta}+\sigma$ and $D=\varphi+\theta+\tau$, so that we obtained

$$
R_{0}=\frac{\eta \frac{\zeta}{\theta}+\sigma}{\varphi+\theta+\tau}
$$




\section{Stability Analysis of the Model Around the Equilibrium Points}

From System (1), the stability of the model will be investigated around the disease-free equilibrium point and disease spread. Because System (1) is a system of nonlinear differential equations, it is first linearized for the equilibrium point $q_{o}=\left(\frac{\zeta}{\theta}, 0,0\right)$, where when $I=0$, and $C=0$ the following Jacobian matrix is obtained.

$$
J_{q_{o}}=\left[\begin{array}{ccc}
-\theta & \frac{-\eta \mu}{\theta} & \xi \\
0 & \frac{-\eta \mu}{\theta}-\varphi-\tau-\theta+\sigma & 0 \\
0 & \tau & -\theta-\xi
\end{array}\right]
$$

The characteristic equation of the Jacobian matrix $J_{q_{o}}$ is determined by equation $\left|J_{q_{o}}-\lambda I\right|=0$ where $I$ is the identity matrix. Thus, the eigen values $\lambda_{1}=-\theta, \lambda_{2}=-\theta-\xi$, and $\lambda_{3}=\frac{-\eta \mu}{\theta}-\varphi-\tau-\theta+\sigma$ with each eigenvalue is negative, the equilibrium point $q_{o}$ is locally asymptotically stable.

Furthermore, for the stability of the model around the equilibrium point of the spread of the disease first linearization of the equation system (1) with the equilibrium point $q_{1}=\left(S^{*}, I^{*}, C^{*}\right)$, is obtained, the following Jacobian matrix $J_{q_{1}}$

$$
\left[\begin{array}{ccc}
-\theta-\frac{\eta(\zeta(\theta+\xi)-\theta(\theta+\xi))\left(\frac{\varphi+\tau+\theta-\sigma}{\eta}\right)}{\varphi+\tau+\theta-\sigma-\xi \tau} & (\varphi+\tau+\theta-\sigma) & \xi \\
\frac{\eta(\zeta(\theta+\xi)-\theta(\theta+\xi))\left(\frac{\varphi+\tau+\theta-\sigma}{\eta}\right)}{\varphi+\tau+\theta-\sigma-\xi \tau} & 0 & 0 \\
0 & \tau & -\theta-\xi
\end{array}\right]
$$

obtained the characteristic equation from the Jacobian matrix $J_{q_{1}}$ as follows.

$$
\begin{aligned}
\lambda^{3}+\lambda^{2}(2 \theta+\xi+P)+\lambda & ((\theta+\xi)(\theta+P)+P M)- \\
& (\xi \tau P+(\theta+\xi) P M)=0
\end{aligned}
$$

where $M=\varphi+\tau+\theta-\sigma$, and $P=$ $\frac{\eta(\zeta(\theta+\xi)-\theta(\theta+\xi))\left(\frac{\varphi+\tau+\theta-\sigma}{\eta}\right)}{\varphi+\tau+\theta-\sigma-\xi \tau}$.

In determining the eigenvalue of equation (3) the calculations performed by Yan-Bin [9] are used by assuming each coefficient of equation (3) with $A=1, B=(2 \theta+\xi+P)$, $C=((\theta+\xi)(\theta+P)+P M)$, and $D=-(\xi \tau P+(\theta+\xi) P M)$, so equation (3) becomes

$$
A \lambda^{3}+B \lambda^{2}+C \lambda-D=0 .
$$

The roots in equation (4) are as follows

$$
\begin{array}{r}
\lambda_{1}=-\frac{1}{3 A}\left(B-\frac{\Pi}{2}+\frac{2\left(3 C A-B^{2}\right)}{\Pi}\right) \\
\lambda_{2}=-\frac{1}{3 A}\left(B+\frac{\Pi}{4}+\frac{\left(3 C A-B^{2}\right)}{\Pi}-\right. \\
\left.\frac{i \sqrt{3}}{2}\left(\frac{\Pi}{2}+\frac{2\left(3 C A-B^{2}\right)}{\Pi}\right)\right) \\
\lambda_{3}=-\frac{1}{3 A}\left(B+\frac{\Pi}{4}+\frac{\left(3 C A-B^{2}\right)}{\Pi}+\right. \\
\left.\frac{i \sqrt{3}}{2}\left(\frac{\Pi}{2}+\frac{2\left(3 C A-B^{2}\right)}{\Pi}\right)\right)
\end{array}
$$

TABLE I: Value of Parameters in Numerical Simulation

\begin{tabular}{c||c}
\hline Parameter & Score \\
\hline \hline$\zeta$ & 0.898986 \\
$\theta$ & 0.004971213 \\
$\eta$ & 0.001505714 \\
$\xi$ & 0.000123 \\
$\varphi$ & 0.000162335 \\
\hline
\end{tabular}

with,

$$
\begin{aligned}
& \Pi=(\Omega+12 \sqrt{3} \sqrt{\Phi})^{\frac{1}{3}} \\
& \Omega=36 C B A-108 D A^{2}-8 B^{3} \\
& \Phi=4 A C^{3}+C^{2} B^{2}-18 C B A D+27 D^{2} A^{2}+4 D B^{3}
\end{aligned}
$$

Next, to determine the eigenvalue of equation (6) so that the real value is negative and stable asymptotically local it is seen that there are two conditions namely when $\Pi>0$ and when $\Pi<0$ which in each condition has the conditions for $\Phi=0$ and $\Phi>0$. With the fulfillment of the given conditions and the terms $B>\frac{\Pi}{2}-\frac{2\left(3 C A-B^{2}\right)}{\Pi}$ or $B>-\frac{\Pi}{4}+\frac{\left(3 C A-B^{2}\right)}{\Pi}$ are met, then taking into account the real part of eigenvalues $\lambda_{i}$, obtained $\operatorname{Re}\left(\lambda_{i}\right)<0, i=1,2,3$. Based on the results obtained, namely $\lambda_{1}<0, \lambda_{2}<0$, and $\lambda_{3}<0$ the equilibrium point of the toxoplasmosis $q_{1}$ epidemic is asymptotically stable.

\section{Bifurcation Analysis}

Bifurcation analysis occurs at the point of equilibrium free of disease and disease infection. Through the conditions of existence and stability at the equilibrium point, it is possible to bifurcate at specified regional boundaries. The bifurcation analysis was carried out on two parameters namely the $\sigma$ parameter and $\tau$ parameter. Several other parameters in System (1) will be of fixed value so that only the parameters used in bifurcation are free. In Table (I), a fixed value is given for each parameter used in the bifurcation analysis of the mathematical model of controlling the toxoplasmosis epidemic.

Based on the conditions of existence and the stability criteria of the equilibrium point for the two parameters; $\sigma$ and $\tau$ by setting the value of the other parameters, a function in the variables $\sigma$ and $\tau$ is formed as follows.

\section{E. Function of Parameters $S_{1,2,3,4}=0$}

From the existence of the equilibrium point in Theorem 1 and the stability criteria for endemic equilibrium points, the following parameter functions are obtained:

For example

$$
\begin{aligned}
& S_{1}=\varphi+\tau+\theta-\sigma=0 \\
& \Longleftrightarrow \varphi+\tau+\theta=\sigma \\
& \Longleftrightarrow 0.000162335+\tau+0.004971213=\sigma \\
& \Longleftrightarrow 0.005134+\tau=\sigma
\end{aligned}
$$

So that the parameter function is obtained in the form $S_{1}=0$ : $0.005134+\tau-\sigma=0$. 


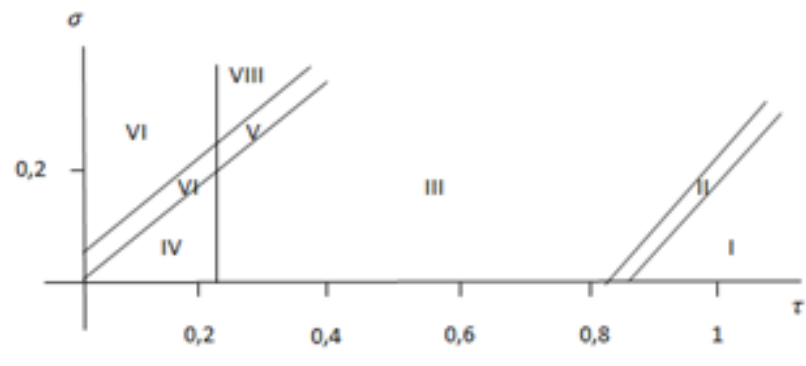

Fig. 2: Bifurcation Diagram Two.

Furthermore, the same method / procedure is performed to calculate the other parameter functions, namely $S_{2}=$ $0: \quad 0.004493285-0.005094 \tau+0.005094 \sigma=0, S_{3}=0:$ $0.88203726-\tau+\sigma=0$, and $S_{4}=0: 0.0000261514+$ $0.004971213 \tau-0.005094 \sigma=0$.

From the parameter function above, it is sketched in the Cartesian plane with $\tau$ as abscissa and $\sigma$ as coordinates. This sketch is called a parameterization diagram or bifurcation diagram with the following illustration.

Figure 2 shows the topological inequality in the area bounded by $S_{1}=0, S_{2}=0, S_{3}=0$, and $S_{4}=0$ by freeing the two parameters $\tau$ and $\sigma$. When the area bounded by $S_{1}=0$, $S_{2}=0, S_{3}=0$, and $S_{4}=0$, so then eight regions are obtained which allow changes in each of the equilibrium points and the stability of the equilibrium points in each region. The dynamics of each region will show the movement of the controlled rate of the spread of toxoplasmosis and the rate of births infected with toxoplasmosis.

\section{Numerical Simulation}

Based on the parameter values given in Table I, the numerical simulation of System (1) in Region I with parameter values $\tau=0.9$ and $\sigma=0.00000983845$ shows that region I has no equilibrium point. The same thing also happened in Region II and III, when given a parameter value $\tau=0.88205013$ and $\sigma=0.000032$ indicates that Region II has no equilibrium point. Similarly, when given a parameter value $\tau=0.65$ and $\sigma=0.006$ shows that Region III has no equilibrium point.

In Region IV, parameter values $\tau=0.271$ and $\sigma=0.00121$ are given. From the calculation of these parameter values show that in Region IV has one equilibrium point that is $q_{o}(179,0,0$,$) . Furthermore, for the stability criteria for the$ equilibrium point $q_{o}$ the value of $\lambda_{1}=-0.0497121, \lambda_{2}=$ -0.18787005 , and $\lambda_{3}=-0.00509421$ show that the stability criteria for the equilibrium point $q_{o}$ is locally asymptotically stable. Numerical simulations for phase portraits in region IV are given in the following figure.

Figure 3 shows that when some initial values are taken around the solution $q_{o}$ it will go to the equilibrium point $q_{o}(179,0,0$,$) , so that the q_{o}$ point is asymptotically stable. The dynamics that can be seen from this condition is the absence of the spread of toxoplasmosis in the Lhokseumawe community.

Furthermore, in the Region V parameter values $\tau=0.27$ and $\sigma=0.06834$ are given. From the calculation of the parameter values, it shows that in the Region $\mathrm{V}$ the equilibrium point changes from the Region $\mathrm{V}$ to the endemic equilibrium point

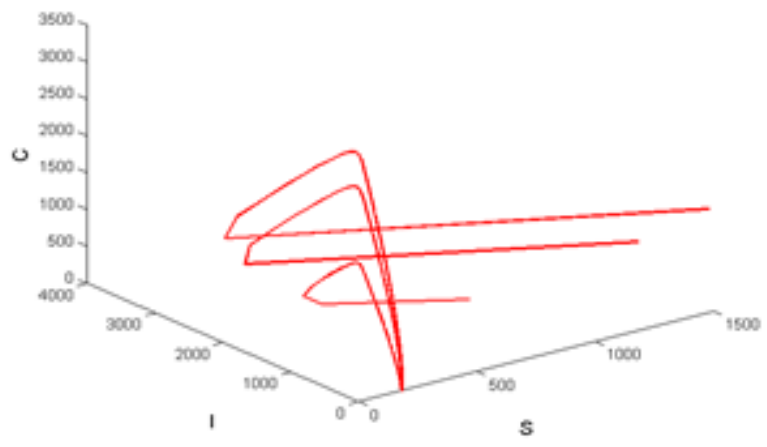

Fig. 3: Portrait of System (1) Phase in Region IV.

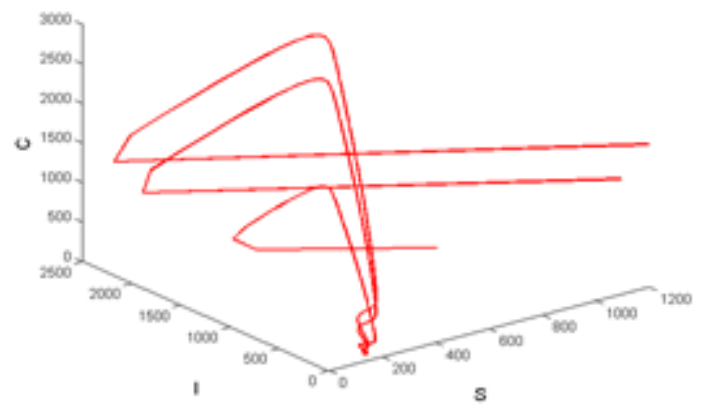

Fig. 4: Portrait of System (1) Phase in Region V.

$q_{1}(134,1,63)$. Furthermore, for the equilibrium point stability criteria $q_{1}$ obtained value $\lambda_{1}=-0.003992, \lambda_{2,3}=-0,003934$, this value indicates that the equilibrium point stability criteria $q_{1}$ is locally asymptotically stable. Numerical simulations for phase portraits in region $\mathrm{V}$ are given in the following figure.

In Fig. 4 it can be shown that for a different initial value when $t$ the solution moves away from the $q_{o}$ point and the solution goes to $q_{1}$. The dynamics that can be seen from this condition is the beginning of the spread of the disease toxoplasmosis in the Lhokseumawe community. This condition is the beginning of the spread of disease in the community environment, it can be shown by the small number of people infected with toxoplasmosis in humans, as many as 1 person. Whereas the controlled population shows that many people are starting to become aware of the spread of toxoplasmosis. This is shown in the number of controlled population of 63 . Thus, in this condition the spread of toxoplasmosis in the environment can be controlled by the community in the face of spreading the disease.

Furthermore, in Region VI the parameter values $\tau=0.063$ and $\sigma=0.05134$ are given. From the calculation of these parameter values show that in Region VI has one endemic equilibrium point $q_{1}(8,83,1032)$. Furthermore, for the equilibrium point stability criteria $q_{1}$ obtained value $\lambda_{1}=-0.129906$, $\lambda_{2,3}=-0.002929$, Furthermore, for the equilibrium point stability criteria $q_{1}$ is locally asymptotically stable. Numerical simulations for phase portraits in area VI are given in the following figure.

In Fig. 5, it can be shown that for a different initial value when $t$ the solution goes to point $q_{1}$. The dynamics that can be seen from this condition is the increase in the 


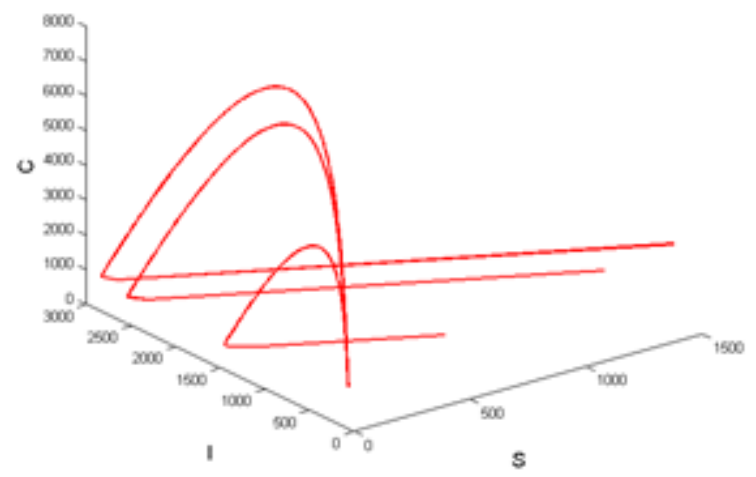

Fig. 5: System Phase 1 portrait in Region VI.

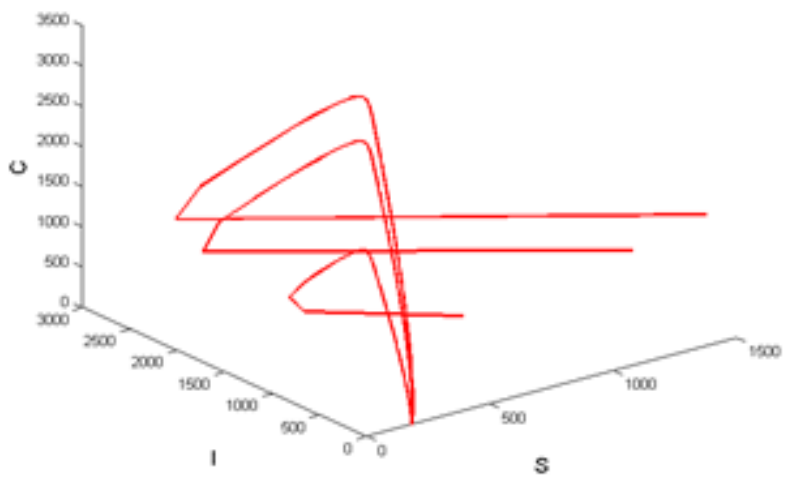

Fig. 6: Portrait of System Phase 1 in Regions VII and III.

spread of toxoplasmosis in the Lhokseumawe community. This condition shows that the spread of disease in the environment is increasingly widespread, resulting in the number of people infected, is amounting to 83 people. This is a very large for an infected environment, because it will have a very dangerous impact on humans in the future. Thus in this condition the handling of disease control must be carried out, one of which is to carry out treatment and socialization related to toxoplasmosis.

Furthermore, in Region VII and Region VIII have the same conditions, if given a parameter value $\tau=0.35$ and $\sigma=0.08704$. From the calculation of these parameter values show that in this Region has a change in the equilibrium point that is from the equilibrium point $q_{1}$ to one equilibrium point $q_{0}$ with a value $q_{0}=(174,0,8)$. Furthermore, for the stability criteria for the equilibrium point $q_{0}$ the value of $\lambda_{1}=-0.00497121, \lambda_{2}=-0.157112$, and $\lambda_{3}=-0.00509421$ shows that the equilibrium point stability $q_{0}$ is locally asymptotically stable. Numerical simulations for phase shots in this area are given in the following figure.

In Fig. 6, it can be shown that for a different initial value when $t$ the solution goes to point $q_{0}$. The dynamics that can be seen from this condition is when there is an increase in the controlled rate, it can suppress the rate of births infected with toxoplasmosis. This can be seen from the number of infected humans at 0 , it means that in this condition toxoplasmosis can be removed from the population (cured). Thus, an increase in the controlled rate is very important to reduce the spread of toxoplasmosis in the Lhokseumawe community.

\section{CONCLUSIONS}

Based on the simulation results above, it is concluded that the mathematical model of the epidemic toxoplasmosis consists of three populations, namely vulnerable populations, infected populations and controlled populations with two equilibrium points, namely disease-free equilibrium points and epidemic toxoplasmosis equilibrium points in the Lhokseumawe City. The results of the mathematical analysis model when the spread of toxoplasmism happened shows that the number of infected populations is increasing and then decreasing, it is triggered by increasing population control, so that it causes the population to be stable. Furthermore, the increase in the number of controlled population gives an indication that treatment has affected the spread of toxoplasmostis as very dangerous to the height of the moon to do the controlling of the spread of the disease, for instance doing the treatment of those who have been infected, following the spread of the disease in the form of the placebo, and changing the type of treatment in the life and the way of life.

Furthermore, from the research done, the research team suggested to look at the effectiveness of drug therapy to influence the spread of toxoplasmosis in the Lhokseumawe city area, so that the dynamics of the toxoplasmosis epidemic occur in every condition.

\section{REFERENCES}

[1] E. Kelting, "Toxoplasma gondii: A mathematical model of its transfer between cats and the environment."

[2] C. Peña, K. Garcia, and H. Martinez, "Hybrid model of the spread of toxoplasmosis between two town of colombia," Tecciencia, vol. 10, no. 18, pp. 1-7, 2015.

[3] M. Hanafiah, M. Kamaruddin, W. Nurcahyo, and Winaruddin, "Studi infeksi toksoplasmosis pada manusia dan hubungannya dengan hewan di banda aceh," Jurnal Kedokteran Hewan, vol. 4, no. 2, pp. 87-92, 2010.

[4] R. Nurcahyo, "Toksoplasmosis pada hewan dan manusia," Yogyakarta: Samudra Biru, 2012.

[5] M. Yanni, "Model matematika penyebaran parasit toksoplasma gondii pada inang perantara dengan interaksi secara tidak langsung oleh inang definitive," Ph.D. dissertation, Universitas Gadjah Mada, 2017.

[6] D. Aranda, R. Villanueva, A. Arenas, and G. González-Parra, "Mathematical modeling of toxoplasmosis disease in varying size populations," Computers \& Mathematics with applications, vol. 56, no. 3, pp. 690696, 2008.

[7] G. González-Parra, A. Arenas, D. Aranda, R. Villanueva, and L. Jódar, "Dynamics of a model of toxoplasmosis disease in human and cat populations," Computers \& Mathematics with applications, vol. 57, no. 10, pp. 1692-1700, 2009.

[8] P. Yongzhen, J. Xuehui, L. Changguo, and G. Shujing, "Dynamics of a model of toxoplasmosis disease in cat and human with varying size populations," Mathematics and Computers in Simulation, vol. 144, pp. $52-59,2018$.

[9] A. Arenas, G. González-Parra, and R.-J. Micó, "Modeling toxoplasmosis spread in cat populations under vaccination," Theoretical population biology, vol. 77, no. 4, pp. 227-237, 2010.

[10] M. Yanni and Z. Zulfahmi, "Analisis pemodelan dan simulasi matematika pengendalian epidemi toksoplasmosis," JTAM (Jurnal Teori dan Aplikasi Matematika), vol. 3, no. 2, pp. 114-120, 2019. 